\title{
Response of Mucocutaneous Lesions to Rituximab in a Case of Melanoma Differentiation Antigen 5-Related Dermatomyositis
}

\author{
Aurélie Clottu $^{\mathrm{a}}$ Emmanuel Laffitte $^{\mathrm{b}}$ Christa Prins $^{\mathrm{b}} \quad$ Carlo Chizzolini $^{\mathrm{a}}$ \\ ${ }^{\mathrm{a}}$ Immunology and Allergy and ${ }^{\mathrm{b}}$ Dermatology and Venereology, University Hospital and School of Medicine, \\ Geneva, Switzerland
}

\section{Key Words}

Dermatomyositis · Amyopathic dermatomyositis - Melanoma differentiation antigen 5 - Clinically amyopathic dermatomyositis $140 \cdot$ Rituximab

\begin{abstract}
We report the first case in Western Europe of a person presenting with dermatomyositis associated with melanoma differentiation antigen 5 antibodies. She sequentially developed severe mucocutaneous erythematous and itchy lesions of the face, scalp, neck, knees and recurrent aphthae. In addition she presented painful, periungual, edematous digital lesions and small ulcers with digital necrosis. Her rapidly evolving, near-fatal interstitial lung disease responded to highdose intravenous cyclophosphamide. However, her recurrent mucocutaneous manifestations improved only after rituximab administration.
\end{abstract}

Copyright $\odot 2013$ S. Karger AG, Basel

\section{Introduction}

Dermatomyositis (DM) is a primary inflammatory disease that typically affects skin and muscles frequently associated with interstitial lung disease (ILD). It en- compasses a spectrum of clinical manifestations in which on the one hand myopathic manifestations (skeletal muscle weakness, and even swallowing and respiratory muscle involvement) may predominate with no or little skin involvement and on the other hand prototypic skin manifestations (heliotrope rash, periorbital edema, and Gottron's sign and papules) may be present with little or no muscular involvement. One extreme end of this spectrum is named clinically amyopathic DM (CADM) or dermatomyositis sine myositis [1]. Several antibodies, termed myositisspecific antibodies, have been associated with exquisitely specific myositic clinical patterns [2]. Such antibodies comprise the anti-aminoacyl-transfer RNA synthetase antibodies, the anti-Mi2, the anti-signal recognition particle (SRP), the anti-small ubiquitin-like modifier activating enzyme, the anti-155/140 (TIF1 $\gamma / \alpha)$, the anti-p140, and the recently identified autoantibody, named anti-CADM-140 also known as anti-melanoma differentiation antigen 5 (MDA5) [3, 4]. Here, we report a case of DM associated with anti-MDA5 antibodies to illustrate the peculiar clinical course of this condition, its response to treatment and in particular to B-cell-depleting therapy.

\section{Case Report}

A 68-year-old woman known for an inflammatory bowel disease currently in remission was hospitalized for erythematous and itchy lesions of the right jaw, scalp, face, neck, knees, and painful periungual erythematous and edematous digital lesions unresponsive to topical corticosteroids. The cutaneous lesions consisted in a diffuse, nonalopecic, scaly dermatosis with erythema on the scalp, widespread erythematous maculopapules of the face involving the upper eyelids, upper back, elbows and the right knee (fig. 1a) without poikiloderma. She presented with fever $\left(38.5^{\circ} \mathrm{C}\right)$, night sweats, loss of appetite, arthralgias, and generalized weakness. She reported severe aphthae, xerostomia, xerophthalmia, and mild Raynaud phenomenon. The muscular strength was normal in the lower limbs, and symmetrically diminished in the upper limbs without proximal predominance. Abnormal laboratory values included: increased C-reactive protein and erythrocyte sedimentation rate, anemia, lymphopenia, hyperferritinemia $(805 \mu \mathrm{g} / \mathrm{l})$, hypoalbuminemia $(27 \mathrm{~g} / \mathrm{l})$ and mild hepatic cytolysis. The creatine kinase and aldolase were within the normal limits. Serologies for cytomegalovirus, EpsteinBarr virus and parvovirus B19 (IgG) re-

\section{KARGER}

E-Mail karger@karger.com

www.karger.com/drm
(C) 2013 S. Karger AG, Base

1018-8665/12/2254-0376\$38.00/0
Carlo Chizzolini, MD

Immunology and Allergy, University Hospital

Rue Gabrielle-Perret-Gentil 4

CH-1211 Geneva 14 (Switzerland)

E-Mail carlo.chizzolini@unige.ch 
vealed previous exposure. An interferon- $\gamma$ releasing assay, human immunodeficiency virus, hepatitis $B$ and $C$ virus and syphilis serology were negative. The sialometry and Schirmer test were pathological. A search for neoplasia performed twice, 2 years apart, was negative and included: an esogastroduodenoscopy, a colonoscopy, a thoracoabdominal CT scan, and a complete gynecological examination with screening for breast cancer. A blood smear performed once at the time of diagnosis was considered exempt of signs of hematological malignancy. An electroneuromyogram of the left deltoid muscle was compatible with myopathic lesions. A biopsy of this muscle showed a mild, perimysial, inflammatory infiltrate. A skin biopsy showed lichenoid interface dermatitis and the immunofluorescence displayed IgM large band granular deposits at the dermoepidermal junction. A capillaroscopy showed unspecific capillary changes. A diagnosis of DM was considered and high-dose prednisone (1 $\mathrm{mg} / \mathrm{kg})$ with a tapering scheme and monthly courses of high-dose intravenous immunoglobulin (IVIG, $3 \mathrm{mg} / \mathrm{kg}$ ) were administered resulting in improved skin lesions, upper limb weakness and arthralgias, but persistence of sicca symptoms.

Four months later, while being on monthly IVIG courses and prednisone (40 $\mathrm{mg} /$ day), in parallel with the resurgence of mild cutaneous lesions, but no articular or muscular complaints, the patient developed fever with cough and an unusual dyspnea (NYHA III) not responding to antibiotics. A thoracic CT scan (fig. 2a) revealed an interstitial infiltrate, ground glass attenuation predominating in the middle and inferior fields associated with subpleural reticulations, and bronchiectases. A surgical lung biopsy was consistent with nonspecific interstitial pneumonia. Pulsed, intravenous cyclophosphamide was initiated while IVIG were discontinued with progressive improvement of her respiratory symptoms. Cyclophosphamide was continued for a total of 9 pulses (total dose: $5.4 \mathrm{~g})$, after which mycophenolate mofetil (MMF, 1-2 g/day) was introduced with low-dose prednisone (10 mg/day) maintenance. A follow-up thoracic CT scan showed the resolution of ground glass attenuation (fig. 2b), paralleled by substantial improvement of the lung function tests (total lung capacity, forced expiration volume at $1 \mathrm{~s}$ and single-breath diffusion of carbon monoxide: 67,49 and $29 \%$ of predicted value 12 days after discharge and 78, 82 and
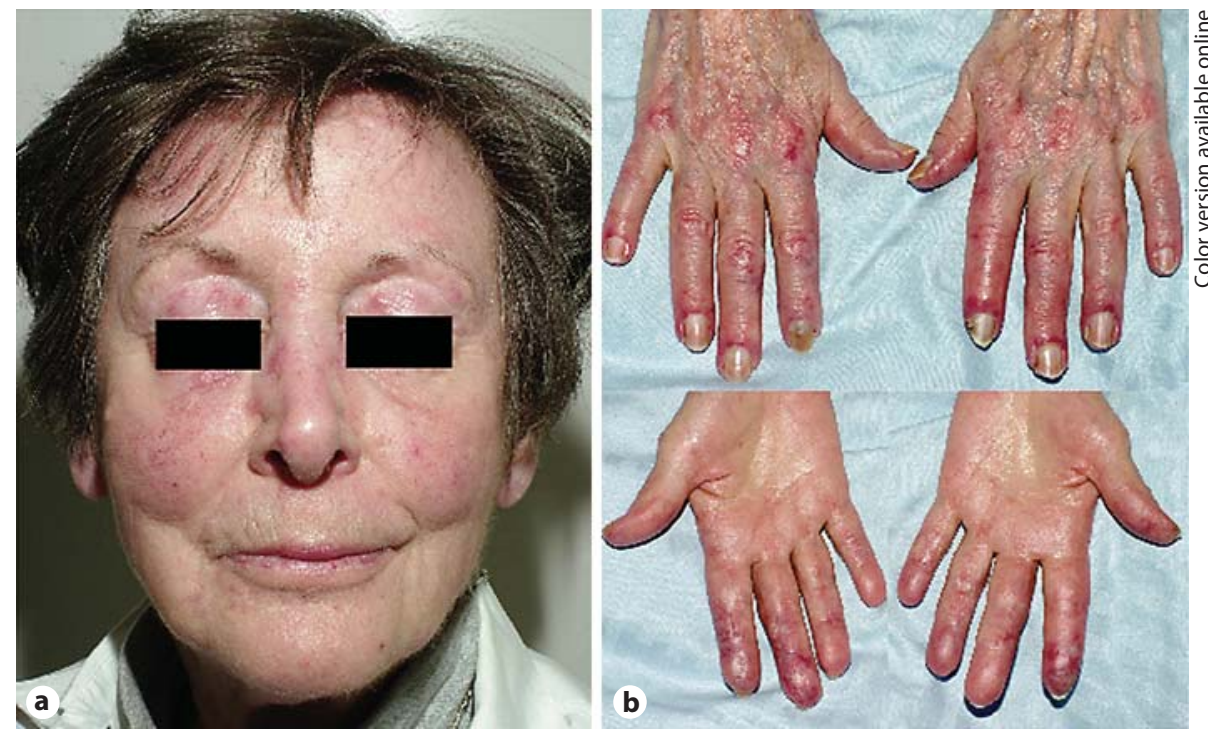

Fig. 1. Typical skin lesions of our MDA5-positive patient before rituximab administration. a Erythematous maculopapules on the jaws and eyelids, refractory to classical treatment. b Erythematous lesions of the metacarpophalangeal articulations, finger tips and the periungual region.
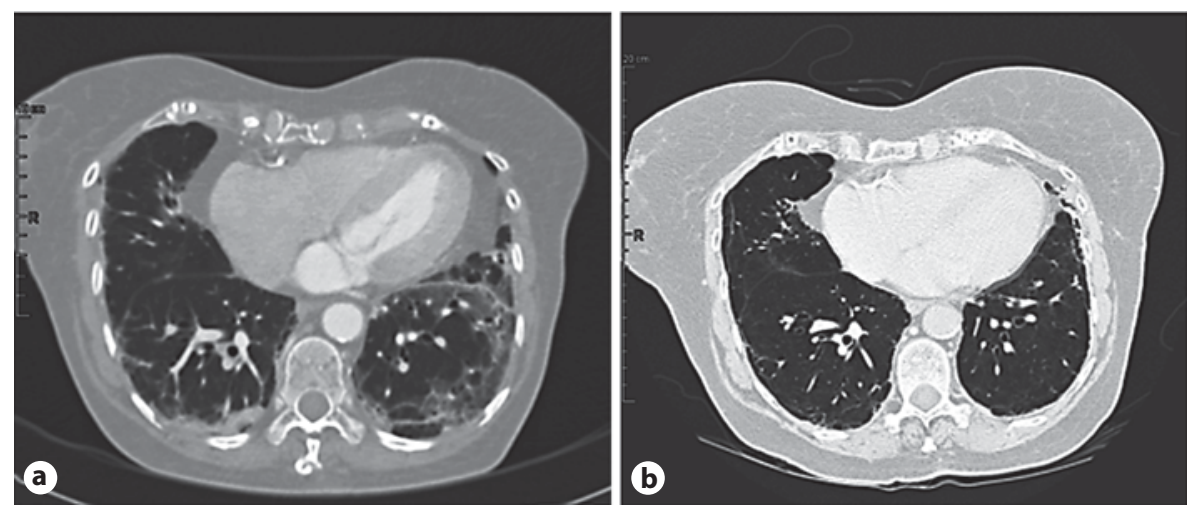

Fig. 2. ILD in our MDA5-related CADM patient. a Before cyclophosphamide administration. b After cyclophosphamide administration.

$49 \%$ one year after discharge). With MMF and prednisone the patient experienced clinical stability during 1 year, but later (2 years after the initiation of DM symptoms), she developed palmar and periungual painful erythematous papules with finger pulp inflammation and discrete superficial necrosis (fig. 1b) accompanied by resurgence of numerous and painful aphthae. These lesions were treated in sequence with topical tacrolimus, IVIG (2 g/ $\mathrm{kg}$ ) and cyclosporine $\mathrm{A}(150 \mathrm{mg} /$ day $)$ in conjunction with MMF (1-2 g/day) with no response. Hydroxychloroquine (400 $\mathrm{mg} /$ day 5 days/week and $200 \mathrm{mg}$ /day 2 days/week) resulted in gastrointestinal pain and diarrhea and was discontinued. A second cycle of intravenous cyclophosphamide consisting in 5 pulses of $600 \mathrm{mg}$ each, resulted in only mild and transient improvement. However, a course of rituximab $(2 \times 1,000 \mathrm{mg} 15$ days apart $)$ allowed a substantial improvement of these mucocutaneous lesions.

Three months later, her second left finger developed an ischemic lesion, which 
rapidly led to pulpar necrosis (fig. 3a). An angio-MRI of the left hand showed an occlusion of the digital artery of the second left finger (fig. 3b). Skin biopsies revealed interface dermatitis, with no obvious vasculitis but with vascular obstruction due to endomyofibrosis. These severe lesions were aggressively treated with iloprost, sildenafil, and an increase in the dose of nifedipine ( $80 \mathrm{mg} /$ day). After 1 month, with the gradual healing of the finger lesions, iloprost and sildenafil were discontinued, and MMF was reintroduced. Afterwards, the patient has remained clinically stable until the writing of this report.

The immunological screening performed on several occasions revealed lowtiter (1/160) anti-nuclear antibody positivity once, intermittently positive SSA antibodies and $\beta_{2}$-glycoprotein I IgG at low to medium titers while the following antibodies were all negative: antineutrophil cytoplasm, antinucleosome, anti-U1RNP, anti-Sm, anti-SSB, antimitochondria, antiLKM, anti-Jo1, anti-PL12, anti-PL7, antiMi2, anti-PM-Scl, anti-SRP, anti-Scl-70, anti-smooth-muscle, antitransglutaminase, and anticardiolipin.

As our patient's symptoms were predominantly cutaneous with a minimal muscular involvement associated with rapidly progressive interstitial pneumonia and pulpar necrosis, we retrospectively searched for anti-MDA5 antibodies and found them positive in 2 of 2 serum samples 2 years apart (at the time of ILD worsening and 2 years later when the skin disease worsened).

\section{Discussion}

We report a case of anti-MDA5 autoantibody-associated DM to illustrate the prototypical clinical evolution of this condition. In addition, for the first time, we document the response to the B-cell-depleting strategy adopted to control its characteristic mucocutaneous lesions.

Our patient presented DM lesions associated with transient muscle abnormalities with little clinical impact. These features are consistent with CADM, reported to account for $10-20 \%$ of DM cases [1]. Alternatively this clinical presentation could be defined as DM with predominant skin involvement. However, the mucocutaneous lesions in our patient had some particularities as she experienced dreadful, recurrent aphthae, associated with painful
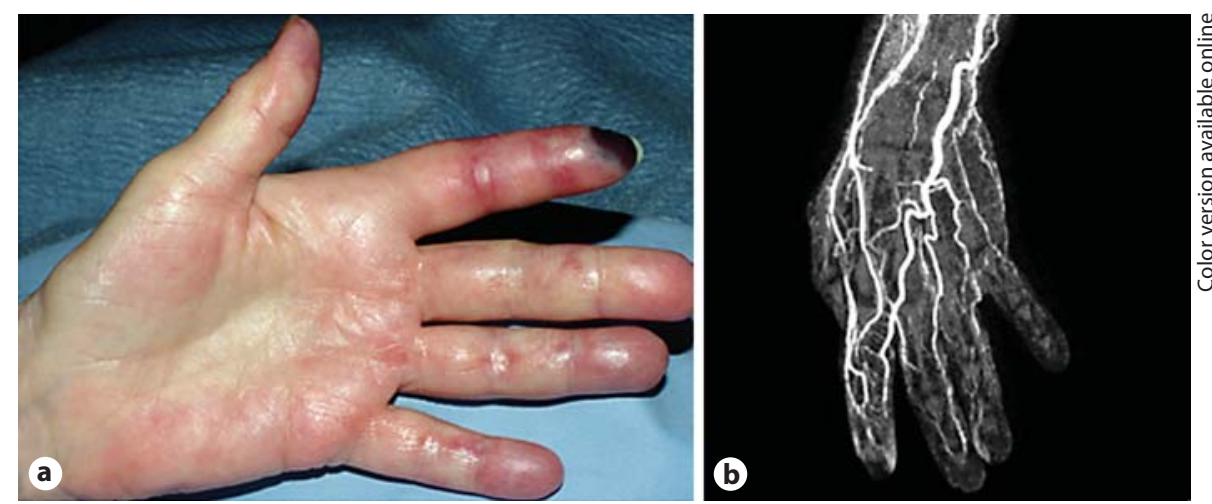

Fig. 3. Digital necrosis in our MDA5 patient 3 years after the onset of CADM. a Pulpar necrosis of the distal phalanx of the second left finger. $\mathbf{b}$ Angio-MRI of the left hand showing obliteration of the second left finger's digital artery, from the distal interphalangeal articulation onwards.

nail fold lesions, skin ulcers especially on the fingers and ears, as well as digital necrosis, which are unusual in classical DM. Fiorentino et al. [5] and Chaisson et al. [6] have described such skin manifestations as characteristic of anti-MDA5-positive patients in conjunction with small and medium vessel vasculopathy, either pauciinflammatory or with mononuclear infiltrates. Consistently with these reports, the skin biopsies in our patient revealed interface dermatitis with vascular obstruction due to endomyofibrosis (confirmed by $a$-actin immunodetection), thickening of the basal membrane, and numerous telangiectasias with no signs of thrombosis or vasculitis. Notably, in our patient, digital necrosis due to macrovascular acute abnormalities occurred 4 months after rituximab administration. This may suggest that B-cell depletion is not efficacious to attenuate the vascular changes observed in MDA5-positive CADM. The possible contribution of an acute thrombotic event superimposing to preexistent vasculopathy cannot be, however, dismissed, and the beneficial effects of rituximab on the mucocutaneous manifestations could have been associated with reduced microvascular disease.

ILD is classically associated with either polymyositis or DM with a prevalence of $5-65 \%$, higher in the antisynthetase subset. Of interest, the rate of ILD is particularly high in CADM patients, with a more severe, corticoresistant, potentially fatal, rapidly progressive course [7]. The antiMDA5 specificity appears to be associated with a prevalence of ILD variably reported between 22 and 93\% [3, 5, 8-11] and with a high mortality, with death rates around $70 \%[10,12]$. In this respect, luckily, our patient responded to a regimen of highdose steroids and pulsed intravenous cyclophosphamide. It should be stressed that most (but not all) reports of rapidly progressive ILD in DM come from Asia, suggesting a genetic or environmental contribution to this aspect of the disease.

Our patient had Raynaud phenomenon and ILD, but no mechanic's hands or polyarthritis. Moreover, she was tested negative for anti-Jo1, anti-PL7 and anti-PL12, making it unlikely that her disease was consistent with antisynthetase syndrome. Furthermore, an extensive search for the presence of neoplasia performed twice was negative in our patient. This is consistent with the absence or low prevalence of neoplastic diseases in anti-MDA5-associated DM [9, 13]. Anti-SSA and Sjögren syndrome are known to be often associated with other connective tissue diseases. Our patient had positive anti-SSA antibodies and experienced sicca symptoms. Curiously, she had fluctuating anti-SSA titers, in parallel with the remissions and recurrences of her disease, which practically is never observed in primary Sjögren syndrome and rarely in systemic lupus erythematosus [14]. The coexistence of MDA5 and SSA antibodies was found in about $30 \%$ of the cases reported by Fiorentino et al. [5]. Finally, the absence in our patient of U1RNP antibody allowed to rule out mixed connective tissue disease.

MDA5 (also known as Helicard or IFIH-1) is a cytoplasmic RNA helicase, the 
levels of which increase in response to type I interferon. It plays an important role in the recognition of double-stranded RNA viruses and mediates the induction of antiviral genes and type I interferon [15]. It has been shown that MDA5 is overexpressed in skin lesions of chronic discoid lupus erythematosus, DM and lichen planus [16]. These data implicate MDA5 in autoimmunity, even if the precise mechanism of its involvement remains unclear. Current hypotheses propose that increased interferon production may play a crucial role in vascular and skin lesions $[17,18]$. Tissue damage would then either result in intracellular antigen release or increase MDA5 expression, resulting in loss of tolerance to MDA5 followed by the production of anti-MDA5 antibody [6, 16, 19]. Alternatively, antiMDA5 autoantibody could be an epiphenomenon during viral infections associated with CADM [10].

Anti-MDA5 seems to be preferentially present in CADM, when searched in patients with connective tissue diseases (polymyositis, systemic lupus erythematosus, systemic sclerosis, CADM, classical DM, cancer-associated DM) or primary ILD [5, $10,13,20]$. In particular, anti-MDA5 was reported in $32.5 \%$ of CADM compared to 9.4\% in DM in one study from China [20] and 65 versus $3 \%$ in another from Japan [13].

Different drug regimens have been attempted to treat CADM and the associated severe, rapidly progressive ILD. Corticosteroids such as prednisone or (methyl) prednisolone are generally used as firstline drugs, with more or less satisfying responses depending on the studies. Hydroxychloroquine has been tried against $\mathrm{DM} / \mathrm{CADM}$ skin lesions with responses that seem to be less satisfying than in other autoimmune conditions [21, 22]. The use of thalidomide, high-dose immunoglobulins or immunosuppressive agents such as MMF, azathioprine, methotrexate, cyclophosphamide, cyclosporine A, oral tacrolimus, or biological agents such as etanercept or infliximab have also been reported with various success. Rituximab has been used in polymyositis, DM and the antisynthetase syndrome in the attempt to control muscle and lung disease, but also predominant skin involvement $[23,24]$. To the best of our knowledge, our case is the first example of response to rituximab, in MDA5related CADM. Cao et al. [20] reported that the severity of the clinical manifestations in MDA5-related DM is in relationship with the titer of anti-MDA5 antibody, implying a direct pathogenic role of this antibody.
Thus, it could be speculated that rituximab may exert its beneficial effects by decreasing the titer of anti-MDA5 antibodies. It cannot be excluded that wider effects through antigen-presenting and cytokineproducing properties of $\mathrm{B}$ cells could be at play.

MDA5-associated DM has been reported in Asia and in the USA [3-5, 8-11, 13, $19,25-31]$. This is the first report of such a condition in Europe. Further studies will be needed to better understand the role of anti-MDA5 antibodies and better exploit the diagnostic value of this autoantibody exquisitely associated with a clinical picture characterized by specific mucocutaneous lesions, severe ILD and necrotic lesions due to vasculopathy.

\section{Acknowledgements}

We are indebted to Dr. Livia CasciolaRosen (Division of Rheumatology, Johns Hopkins University School of Medicine, Baltimore, Md., USA) for kindly assessing the presence of anti-MDA5 antibodies in the serum of our patient. This work was supported in part by a Swiss National Science Foundation grant to C.C. (310030_ 140791).

\section{References}

1 Sontheimer RD: Would a new name hasten the acceptance of amyopathic dermatomyositis (dermatomyositis sine myositis) as a distinctive subset within the idiopathic inflammatory dermatomyopathies spectrum of clinical illness? J Am Acad Dermatol 2002; 46:626-636.

12 Casciola-Rosen L, Mammen AL: Myositis autoantibodies. Curr Opin Rheumatol 2012;24: 602-608.

-3 Sato S, Hirakata M, Kuwana M, Suwa A, Inada S, Mimori T, Nishikawa T, Oddis CV, Ikeda Y: Autoantibodies to a $140-\mathrm{kDa}$ polypeptide, CADM-140, in Japanese patients with clinically amyopathic dermatomyositis. Arthritis Rheum 2005;52:1571-1576.

4 Sato S, Hoshino K, Satoh T, Fujita T, Kawakami Y, Kuwana M: RNA helicase encoded by melanoma differentiation-associated gene 5 is a major autoantigen in patients with clinically amyopathic dermatomyositis: association with rapidly progressive interstitial lung disease. Arthritis Rheum 2009;60:2193-2200.

5 Fiorentino D, Chung L, Zwerner J, Rosen A, Casciola-Rosen L: The mucocutaneous and systemic phenotype of dermatomyositis patients with antibodies to MDA5 (CADM-
140): a retrospective study. J Am Acad Dermatol 2011;65:25-34.

6 Chaisson NF, Paik J, Orbai AM, CasciolaRosen L, Fiorentino D, Danoff S, Rosen A: A novel dermato-pulmonary syndrome associated with MDA-5 antibodies: Report of 2 cases and review of the literature. Medicine (Baltimore) 2012;91:220-228.

7 Mimori T, Nakashima R, Hosono Y: Interstitial lung disease in myositis: clinical subsets, biomarkers, and treatment. Curr Rheumatol Rep 2012;14:264-274.

-8 Fujikawa K, Kawakami A, Kaji K, Fujimoto M, Kawashiri S, Iwamoto N, Aramaki T, Ichinose K, Tamai M, Kamachi M, Nakamura $\mathrm{H}$, Ida $\mathrm{H}$, Origuchi T, Ishimoto $\mathrm{H}$, Mukae $\mathrm{H}$, Kuwana M, Kohno S, Takehara K, Sato S, Eguchi K: Association of distinct clinical subsets with myositis-specific autoantibodies towards anti-155/140-kDa polypeptides, anti-140-kDa polypeptides, and anti-aminoacyl tRNA synthetases in Japanese patients with dermatomyositis: a single-centre, cross-sectional study. Scand J Rheumatol 2009;38:263-267.

-9 Nakashima R, Imura Y, Kobayashi S, Yukawa $\mathrm{N}$, Yoshifuji H, Nojima T, Kawabata D, Ohmura K, Usui T, Fujii T, Okawa K, Mimori T:
The RIG-I-like receptor IFIH1/MDA5 is a dermatomyositis-specific autoantigen identified by the anti-CADM-140 antibody. Rheumatology (Oxford) 2010;49:433-440.

-10 Koga T, Fujikawa K, Horai Y, Okada A, Kawashiri SY, Iwamoto N, Suzuki T, Nakashima Y, Tamai M, Arima K, Yamasaki S, Nakamura H, Origuchi T, Hamaguchi Y, Fujimoto M, Ishimatsu Y, Mukae H, Kuwana M, Kohno S, Eguchi K, Aoyagi K, Kawakami A: The diagnostic utility of anti-melanoma differentiationassociated gene 5 antibody testing for predicting the prognosis of Japanese patients with DM. Rheumatology (Oxford) 2012;51:1278-1284.

-11 Hamaguchi Y, Kuwana M, Hoshino K, Hasegawa M, Kaji K, Matsushita T, Komura K, Nakamura M, Kodera M, Suga N, Higashi A, Ogusu K, Tsutsui K, Furusaki A, Tanabe H, Sasaoka S, Muro Y, Yoshikawa M, Ishiguro N, Ayano M, Muroi E, Fujikawa K, Umeda Y, Kawase M, Mabuchi E, Asano Y, Sodemoto K, Seishima M, Yamada H, Sato S, Takehara $\mathrm{K}$, Fujimoto M: Clinical correlations with dermatomyositis-specific autoantibodies in adult Japanese patients with dermatomyositis: a multicenter cross-sectional study. Arch Dermatol 2011;147:391-398. 
12 Kang EH, Lee EB, Shin KC, Im CH, Chung $\mathrm{DH}$, Han SK, Song YW: Interstitial lung disease in patients with polymyositis, dermatomyositis and amyopathic dermatomyositis. Rheumatology (Oxford) 2005;44:1282-1286.

$\checkmark 13$ Hoshino K, Muro Y, Sugiura K, Tomita Y, Nakashima R, Mimori T: Anti-MDA5 and anti-TIF1-gamma antibodies have clinical significance for patients with dermatomyositis. Rheumatology (Oxford) 2010;49:17261733.

14 Wahren M, Tengner P, Gunnarsson I, Lundberg I, Hedfors E, Ringertz NR, Pettersson I: Ro/SS-A and La/antibody level variation in patients with Sjögren's syndrome and systemic lupus erythematosus. J Autoimmun 1998; 11:29-38.

15 Barral PM, Sarkar D, Su ZZ, Barber GN, DeSalle R, Racaniello VR, Fisher PB: Functions of the cytoplasmic RNA sensors RIG-I and MDA-5: key regulators of innate immunity. Pharmacol Ther 2009;124:219-234.

16 Zahn S, Barchet W, Rehkamper C, Hornung T, Bieber T, Tuting T, Wenzel J: Enhanced skin expression of melanoma differentiationassociated gene 5 (MDA5) in dermatomyositis and related autoimmune diseases. J Am Acad Dermatol 2011;64:988-989.

17 Wenzel J, Tuting T: An IFN-associated cytotoxic cellular immune response against viral, self-, or tumor antigens is a common pathogenetic feature in 'interface dermatitis'. J Invest Dermatol 2008;128:2392-2402.

18 Trinchieri G: Type I interferon: friend or foe? J Exp Med 2010;207:2053-2063.
19 Sato S, Kuwana M: Clinically amyopathic dermatomyositis. Curr Opin Rheumatol 2011; 22:639-643.

20 Cao H, Pan M, Kang Y, Xia Q, Li X, Zhao X, Shi R, Lou J, Zhou M, Kuwana M, Ding X, Zheng J: Clinical manifestations of dermatomyositis and clinically amyopathic dermatomyositis patients with positive expression of anti-MDA5 antibody. Arthritis Care Res (Hoboke) 2012;64:1602-1610.

21 Veetil BM, Whitt I: Amyopathic dermatomyositis presenting as skin necrosis. J Clin Rheumatol 2011;17:332-333.

22 Cao H, Parikh TN, Zheng J: Amyopathic dermatomyositis or dermatomyositis-like skin disease: retrospective review of 16 cases with amyopathic dermatomyositis. Clin Rheumatol 2009;28:979-984.

23 Joshi N, Nautiyal A, Davies PG: Successful use of rituximab in recalcitrant skin predominant dermatomyositis. J Clin Rheumatol 2011;17: 111-112.

24 Dinh HV, McCormack C, Hall S, Prince HM: Rituximab for the treatment of the skin manifestations of dermatomyositis: a report of 3 cases. J Am Acad Dermatol 2007;56:148-153.

25 Chinoy H, Fertig N, Oddis CV, Ollier WE, Cooper RG: The diagnostic utility of myositis autoantibody testing for predicting the risk of cancer-associated myositis. Ann Rheum Dis 2007;66:1345-1349.
26 Gono T, Kawaguchi Y, Satoh T, Kuwana M, Katsumata Y, Takagi K, Masuda I, Tochimoto A, Baba S, Okamoto Y, Ota Y, Yamanaka H: Clinical manifestation and prognostic factor in anti-melanoma differentiation-associated gene 5 antibody-associated interstitial lung disease as a complication of dermatomyositis. Rheumatology (Oxford) 2010;49:1713-1719.

27 Gunawardena H, Betteridge ZE, McHugh NJ Myositis-specific autoantibodies: their clini$\mathrm{cal}$ and pathogenic significance in disease expression. Rheumatology (Oxford) 2009;48: 607-612.

28 Ikeda N, Takahashi K, Yamaguchi Y, Inasaka M, Kuwana M, Ikezawa Z: Analysis of dermatomyositis-specific autoantibodies and clinical characteristics in Japanese patients. J Dermatol 2011;38:973-979.

29 Kang EH, Nakashima R, Mimori T, Kim J, Lee YJ, Lee EB, Song YW: Myositis autoantibodies in Korean patients with inflammatory myositis: anti-140-kDa polypeptide antibody is primarily associated with rapidly progressive interstitial lung disease independent of clinically amyopathic dermatomyositis. BMC Musculoskelet Disord 2010;11:223.

30 Muro Y, Sugiura K, Hoshino K, Akiyama M, Tamakoshi K: Epidemiologic study of clinically amyopathic dermatomyositis and antimelanoma differentiation-associated gene 5 antibodies in central Japan. Arthritis Res Ther 2011;13:R214.

-31 Sato S, Kuwana M, Fujita T, Suzuki Y: Amyopathic dermatomyositis developing rapidly progressive interstitial lung disease with elevation of anti-CADM-140/MDA5 autoantibodies. Mod Rheumatol 2012;22:625-629. 